\title{
Closed-Form Design of All-Pass Fractional Delay Filters
}

\author{
Soo-Chang Pei, Fellow, IEEE, and Peng-Hua Wang
}

\begin{abstract}
In this letter, we propose a novel all-pass (AP) fractional delay filter whose denominator polynomial is obtained by truncating the power series of a certain function. This function is derived from the frequency response of the denominator whose magnitude response is related to the desired phase response through the Hilbert transform since the denominator of a stable AP filter is of minimum phase. The target function and corresponding power series are calculated analytically and expressed in closed form. The closed-form expressions facilitate the analysis of stability. According to the properties for the coefficients of the denominator polynomial, we show that the proposed AP filter is stable for positive delay. Numerical examples indicate that the phase delays of the proposed filters are flat around DC.
\end{abstract}

Index Terms-Allpass filter, fractional delay filter, minimum phase, phase delay.

\section{INTRODUCTION}

D IGITAL implementation of fractional delay (FD) occurs in many applications such as sound synthesis and timing adjustment in digital receivers. Therefore, design of digital FD filters is important and has been widely studied and reported in the literature [1]. Several closed-form FD filters are investigated because there exist efficient and tunable structures for implementation. Finite-impulse response (FIR) FD filters with closed-form coefficients can be derived by windowing their ideal impulse responses [1], [2], by solving the Vandermonde system [3], or by expanding a certain function to power series [4]. The Vandermonde FIR FD filters that are identical to the series expansion have maximally flat (MF) frequency responses [4]. The MF FIR FD filters can be implemented in module [4] or in tunable structures such as the Farrow structure [1].

All-pass (AP) filters are a natural choice to design the FD filters, since the AP filters have unity magnitude responses within the whole frequency band structurally. However, it is necessary for AP filters to check their stability, which is guaranteed for the FIR filters. The methods of AP filter design can be surely applied to the FD filters [5]. Like the FIR MF FD filters, closed-form AP FD filters whose coefficients are obtained by solving the Vandermonde system has MF phase delay [6]. There exist tunable structures for the MF AP FD filters [7]. By over-sampling

Manuscript received January 6, 2003; revised March 20, 2004. This work was supported by the National Science Council, Taiwan, R.O.C., under Contracts NSC 91-2219-E-002-044 and NSC 93-2752-E—002-006-PAE. The associate editor coordinating the review of this manuscript and approving it for publication was Dr. Richard C. Kavanagh.

The authors are with the Department of Electrical Engineering, National Taiwan University, Taipei, Taiwan, R.O.C. (e-mail: pei@cc.ee.ntu.edu.tw).

Digital Object Identifier 10.1109/LSP.2004.835473 input signal, an efficient implementation of wideband FD filters is proposed in [8].

In this letter, we proposed a new AP FD filter with closedform coefficients by series expansion. The phase delay of the denominator is calculated from the desired overall delay and accordingly the magnitude response can be derived through the Hilbert transform based on the fact that the denominator is a minimum phase polynomial. The ideal transfer function of the denominator can be derived by the magnitude and the phase responses. The denominator polynomials of the AP filters are obtained by truncating the power series of the ideal transfer function. Stability checked by a theorem about the bound of the zeros of a polynomial is guaranteed for positive delay.

\section{FREQUENCY RESPONSE OF DENOMINATOR}

The transfer function of an $N$ th-order real-coefficient AP filter is represented by

$$
H(z)=\frac{a_{N}+a_{N-1} z^{-1}+\cdots+a_{0} z^{-N}}{a_{0}+a_{1} z^{-1}+\cdots+a_{N} z^{-N}}=\frac{z^{-N} A\left(z^{-1}\right)}{A(z)}
$$

where the numerator is the mirror-image polynomial of the denominator. Although we usually let $a_{0}=1$ for AP filters to prevent the null solution, in this letter we do not make such an assumption, so as to facilitate our derivation and discussion. The phase response of the AP filter can be expressed by

$$
\arg \left[H\left(e^{j \omega}\right)\right]=-N \omega-2 \arg \left[A\left(e^{j \omega}\right)\right]
$$

where $\arg \left[A\left(e^{j \omega}\right)\right]$ is the phase response of the denominator. Given the desired phase response $P(\omega)$, we want to find a set of coefficients $a_{n}$ 's so that $\arg \left[H\left(e^{j \omega}\right)\right] \approx P(\omega)$, or equivalently

$$
\arg \left[A\left(e^{j \omega}\right)\right] \approx-\frac{1}{2}[N \omega+P(\omega)] .
$$

Suppose the desired phase response for the $N$ th-order AP FD filter is

$$
P(\omega)=-(N+d) \omega
$$

where $d$, restricted by $-1<d<1$ in this letter, is the fractional part of the delay. Then (3) gives the desired phase response of the denominator

$$
\arg \left[A\left(e^{j \omega}\right)\right] \approx \frac{1}{2} d \omega .
$$

We assume that the magnitude response $\left|A\left(e^{j \omega}\right)\right|$ approximates a minimum phase system with the phase response expressed by (5). The magnitude $\left|A\left(e^{j \omega}\right)\right|$ is related to the phase response 
$\arg \left[A\left(e^{j \omega}\right)\right]$ by the discrete Hilbert transformation [2]. Specifically speaking

$$
\ln \left|A\left(e^{j \omega}\right)\right|=C+\frac{1}{2 \pi} P \int_{-\pi}^{\pi} \arg \left[A\left(e^{j \theta}\right)\right] \cot \left(\frac{\omega-\theta}{2}\right) d \theta
$$

where the symbol $P$ denotes the Cauchy principal value of the integral. The constant $C$ in (6) is calculated by

$$
C=\frac{1}{2 \pi} \int_{-\pi}^{\pi} \ln \left|A\left(e^{j \omega}\right)\right| d \omega .
$$

Since $C$ represents a scaling factor to $\left|A\left(e^{j \omega}\right)\right|$, it will be canceled out in the case of the AP filter. Hence, we let $C=0$ without loss of generality.

Substituting (5) into (6), we may express $\left|A\left(e^{j \omega}\right)\right|$ in closed form. By means of the Leibniz's theorem for differentiation of an integral [9], it is easy to calculate the integral of (6).

Property 1: The magnitude response of a minimum phase system with phase response $\omega d / 2$ is

$$
\left|A\left(e^{j \omega}\right)\right|=(2+2 \cos \omega)^{-d / 2} .
$$

Proof: Let

$$
f(\omega)=\frac{1}{2 \pi} \int_{-\pi}^{\pi} \theta \cot \left(\frac{\omega-\theta}{2}\right) d \theta .
$$

We obtain that $\ln \left|A\left(e^{j \omega}\right)\right|=f(\omega) \times d / 2$ after substituting (5) into (6). By the Leibniz's theorem, we have

$$
\begin{aligned}
\frac{\mathrm{d}}{\mathrm{d} \omega} f(\omega) & =\frac{1}{2 \pi} \int_{-\pi}^{\pi} \theta\left[\frac{\partial}{\partial \omega} \cot \left(\frac{\omega-\theta}{2}\right)\right] \mathrm{d} \theta \\
& =\frac{1}{2 \pi} \int_{-\pi}^{\pi} \theta\left[-\frac{1}{2} \csc ^{2}\left(\frac{\omega-\theta}{2}\right)\right] \mathrm{d} \theta=\tan \frac{1}{2} \omega .
\end{aligned}
$$

Therefore

$$
f(\omega)=K+\int \tan \frac{1}{2} \omega d \omega=K-\ln \cos ^{2} \frac{1}{2} \omega
$$

where $K$ is a constant. To determine $K$, we let $\omega=0$, equate (9) with (10), and obtain that

$$
K=f(0)=\frac{1}{2 \pi} \int_{-\pi}^{\pi} \theta \cot \left(-\frac{1}{2} \theta\right) d \theta=-\ln 4 .
$$

Therefore, $\ln \left|A\left(e^{j \omega}\right)\right|=-(1 / 2) d \ln \left[4 \cos ^{2}((1 / 2) \omega)\right]$ and the magnitude response can be obtained and expressed as (8).

Since the magnitude response and the phase response of the denominator are obtained, the frequency response of the denominator is calculated by the product of the magnitude and the phase, and the transfer function can be obtained easily. This transfer function could be regarded as the ideal transfer function of the AP FD filter. We obtain the following property.

Property 2: The ideal transfer function of the denominator for an $N$ th-order AP FD filter with total delay of $N+d$ is

$$
A_{\text {ideal }}(z)=\left(1+z^{-1}\right)^{-d} .
$$

Remark: In fact, the denominators of Thiran's MF AP FD filters converge to $A_{\text {ideal }}(z)$ as $N$ approaches infinity. By expressing the coefficients of Thiran's MF AP FD filters as

$$
a_{m}=\frac{(-1)^{m}(d)_{m}}{(N+d+1)_{m}}\left(\begin{array}{l}
N \\
m
\end{array}\right)
$$

we have

$$
\begin{aligned}
a_{m} & =\frac{(-1)^{m}(d)_{m}}{(N+d+1)_{m}}\left(\begin{array}{c}
N \\
m
\end{array}\right) \\
& =\frac{(-1)^{m}(d)_{m}}{m !} \times \frac{N}{N+d+1} \times \frac{N-1}{N+d+2} \times \cdots \times \frac{N-m+1}{N+d+m}
\end{aligned}
$$

and

$$
\begin{aligned}
\lim _{N \rightarrow \infty} a_{m} & =\frac{(-1)^{m}(d)_{m}}{m !} \\
& =\frac{(-d) \times(-d-1) \times \cdots \times(-d-m+1)}{m !}=\left(\begin{array}{c}
-d \\
m
\end{array}\right)
\end{aligned}
$$

where $\left(\begin{array}{c}-d \\ m\end{array}\right)$ is the coefficients of the Taylor's series of $A_{\text {ideal }}(z)$ expanded at $z^{-1}=0$, and $(x)_{n}$ is the Pochhammer's symbol defined by $(x)_{0}=1$ and $(x)_{n}=x \times(x+1) \times \cdots \times(x+n-1)$.

Based on the magnitude response expressed by (8) or the ideal transfer function of (11), we may find the coefficients of the denominator $A(z)$ by well-developed methods for FIR filter design. In this letter, we will expand (11) into its power series to obtain the closed-form formulas for the coefficients directly.

Property 3: The transfer function of the denominator of an $N$ th-order AP FD filter can be expressed by

$$
A(z)=\sum_{n=0}^{\infty} \frac{(d)_{n}}{n !}\left(\frac{1-z^{-1}}{2}\right)^{n} .
$$

Proof: This result can be obtained by expanding the ideal transfer function around $z=1$. Expressing $A_{\text {ideal }}(z)$ in (11) as $2^{-d}\left[\left(1+\left(z^{-1}-1\right) / 2\right)\right]^{-d}$, expanding it as the binomial series, and truncating the resulting series up to the first $N+1$ terms, we obtain (14), except for the scaling factor of $2^{-d}$. The factor can be dropped since it will be cancelled out in the AP transfer function.

According to Property 3 , it is easy to obtain the filter coefficients $a_{n}$ 's in closed form by expanding the right side of (14) and then collecting terms of the same power. After some algebraic manipulations, the coefficients in (1) can be expressed as

$$
a_{n}=\frac{(-1)^{n}}{n ! 2^{n}} \sum_{k=0}^{N-n} \frac{(d)_{k+n}}{k ! 2^{k}}
$$

for $n=0,1, \ldots, N$.

The coefficients of the $N$ th-order AP FD filter are now obtained in closed form. Although we derive the coefficients of the filter according to the assumption of minimum phase system for the denominator, this assumption does not guarantee stability of $A(z)$ in (14) because of truncation of series. In Section III, we will discuss the stability of the proposed AP filter. 


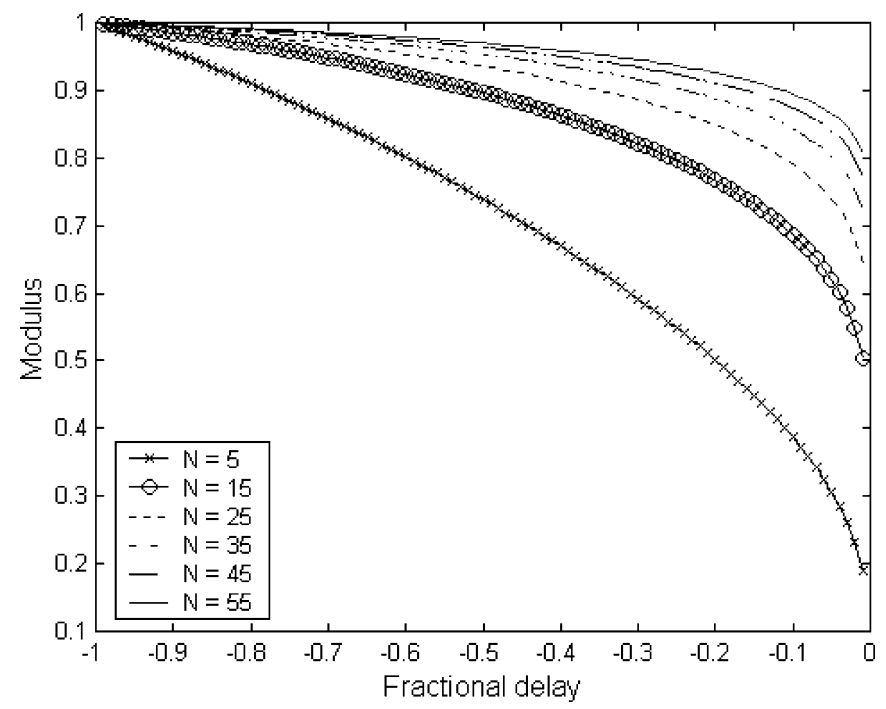

Fig. 1. Plot of largest poles in modulus for $-1<d<0$. Because we give a proof of stability only for $0<d<1$, the stability for $-1<d<0$ is illustrated by numerically computing the poles and by showing that their moduli are less than unity.

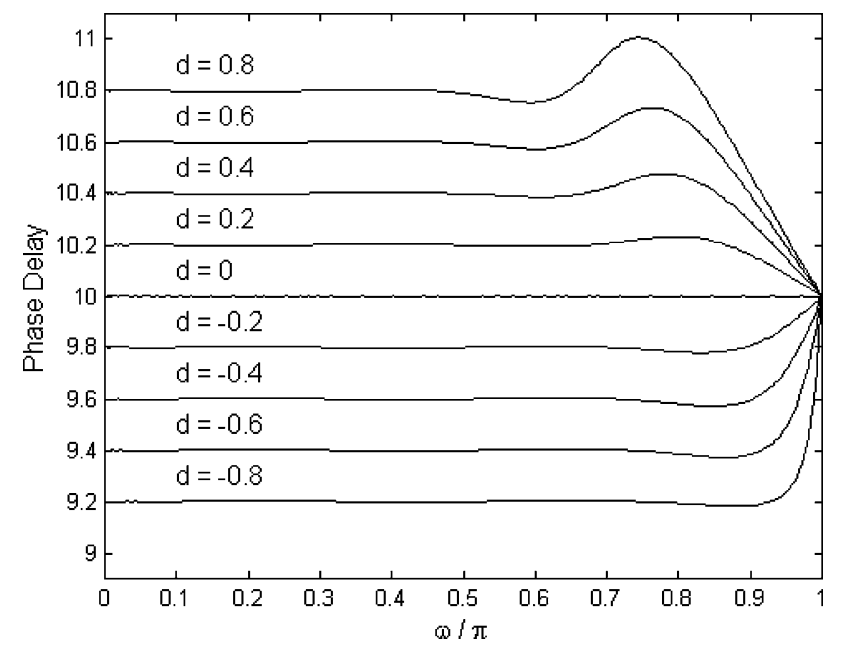

Fig. 2. Phase delay plots for $N=10$. These plots suggest that the bandwidths for $d<0$ are wider than bandwidths for $d>0$.

\section{Stability AND Design Results}

To test the stability of the proposed AP filter, we may apply the Schur-Cohn criterion or the more efficient Jury-Marden criterion [10]. Nevertheless, it is difficult to evaluate the Schur determinants because the summation in (14) can not be simplified furthermore. It is also complicated to establish the Jury-Marden arrays for the same reason. Therefore, we have to find another way to test the stability. In this letter, we will apply the Eneström-Kakeya theorem [11], [12] stated as the following.

Theorem 1: Let $p(x)=\sum_{n=0}^{N} a_{n} x^{N-n}, N \geq 1$, be a polynomial with $a_{n}>0$ for $0 \leq n \leq N$. Let $r_{n}=a_{n+1} / a_{n}$ for $0 \leq n<N$. Then all the zeros of $p(x)$ are contained in the annulus

$$
\min _{n} r_{n} \leq|x| \leq \max _{n} r_{n}
$$

By using the closed-form coefficients in (14), we can show that the coefficients are decreasing in modulus. In fact, we have the following property.

Property 4: The denominator polynomial $A(z)$ can be represented as

$$
A(z)=h_{0}-h_{1} z^{-1}+h_{2} z^{-2}-\cdots+(-1)^{N} h_{N} z^{-N}
$$

for $0<d<1$ where $h_{0}>h_{1}>h_{2}>\cdots>h_{N}>0$.

Proof: It is obvious that $h_{n}=(-1)^{n} a_{n}>0$ for $n=$ $0,1, \ldots, N$. Since $h_{n}$ can be rewritten as

$$
\begin{aligned}
h_{n} & =\sum_{k=0}^{N-n} \frac{(d)_{k+n}}{n ! k ! 2^{n+k}}=\frac{(d)_{n}}{n ! 2^{n}}+\sum_{k=1}^{N-n} \frac{(d)_{k+n}}{n ! k ! 2^{n+k}} \\
& =\frac{(d)_{n}}{n ! 2^{n}}+\sum_{k=0}^{N-n-1} \frac{(d)_{k+n+1}}{n !(k+1) ! 2^{n+k+1}}
\end{aligned}
$$

we have

$$
\begin{aligned}
h_{n+1}-h_{n} & =-\frac{(d)_{n}}{n ! 2^{n}}+\sum_{k=1}^{N-n} \frac{(k-n-1)(d)_{k+n}}{(n+1) ! k ! 2^{k+n}} \\
& <-\frac{(d)_{n}}{n ! 2^{n}}+\sum_{k=1}^{\infty} \frac{(k-n-1)(d)_{k+n}}{(n+1) ! k ! 2^{k+n}} \\
& =\frac{2^{n+d}(d-1)_{n+1}}{(n+1) !}<0
\end{aligned}
$$

for $N-n+1 \geq n-1$. That is, $h_{n+1}<h_{n}$ for $2 n \leq N$. On the other hand, to prove $h_{n+1}<h_{n}$ for $2 n>N$, we first express $h_{n}$ as $h_{n}=\sum_{k=0}^{N-n} \alpha_{k, n}$ where $\alpha_{k, n}=(d)_{k+n} /\left(n ! k ! 2^{n+k}\right)$. Since $k \leq N-n, 2 n>N$ and $d<1$, we have

$$
\frac{\alpha_{k, n+1}}{\alpha_{k, n}}=\frac{n+k+d}{2 n+2} \leq \frac{N+d}{2 n+2}<\frac{N+d}{N+2}<1
$$

and, finally

$$
\begin{aligned}
\frac{h_{n+1}}{h_{n}} & =\frac{\alpha_{0, n+1}+\alpha_{1, n+1}+\cdots+\alpha_{N-n-1, n+1}}{\alpha_{0, n}+\alpha_{1, n}+\cdots+\alpha_{N-n-1, n}+\alpha_{N-n, n}} \\
& <\frac{\alpha_{0, n}+\alpha_{1, n}+\cdots+\alpha_{N-n-1, n}}{\alpha_{0, n}+\alpha_{1, n}+\cdots+\alpha_{N-n-1, n}+\alpha_{N-n, n}}<1 .
\end{aligned}
$$

Based on Property 4, it is easy to show that the proposed AP filter is stable for $0<d<1$. Let $\hat{A}(z)=A(-z)$. Since the coefficients of $\hat{A}(z)$ are positive and strictly monotone decreasing, the zeros of $\hat{A}(z)$ lie in $|z|<1$ by Theorem 1 . Therefore the zeros $A(z)$ also lie in $|z|<1$. We conclude that the AP FD filter with denominator $A(z)$ is stable.

We do not prove the stability of the proposed filters for $-1<$ $d<0$ in this letter. However, by numerically computing the zeros of $A(z)$, the largest moduli of zeros are less than unity for $N<100$. Fig. 1 shows the plot of largest moduli for $N=$ $5,15,25,35,45,55$, and $-1<d<0$. The pole of largest modulus for $N=55$ and $d=-0.99$ is a negative real pole of -0.99963284345625 whose modulus is near but less than unity.

As an illustration of the proposed filters, Fig. 2 shows the design results of the tenth-order AP FD filters. Fig. 2 is the plot of the phase delays versus normalized frequency for $d=$ $-0.8,-0.6, \ldots, 0.6,0.8$. This plot suggests that the bandwidths 


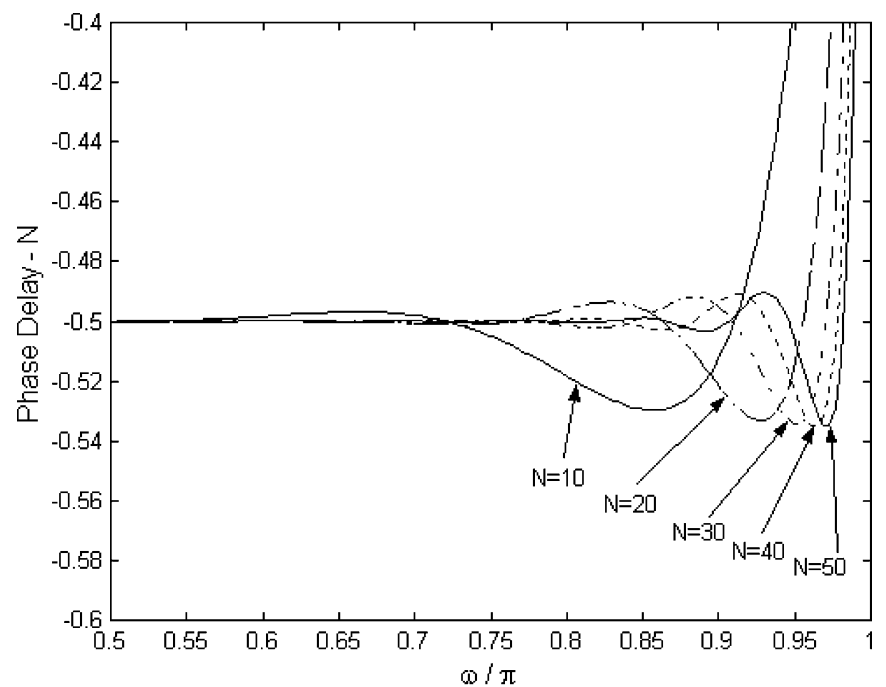

(a)

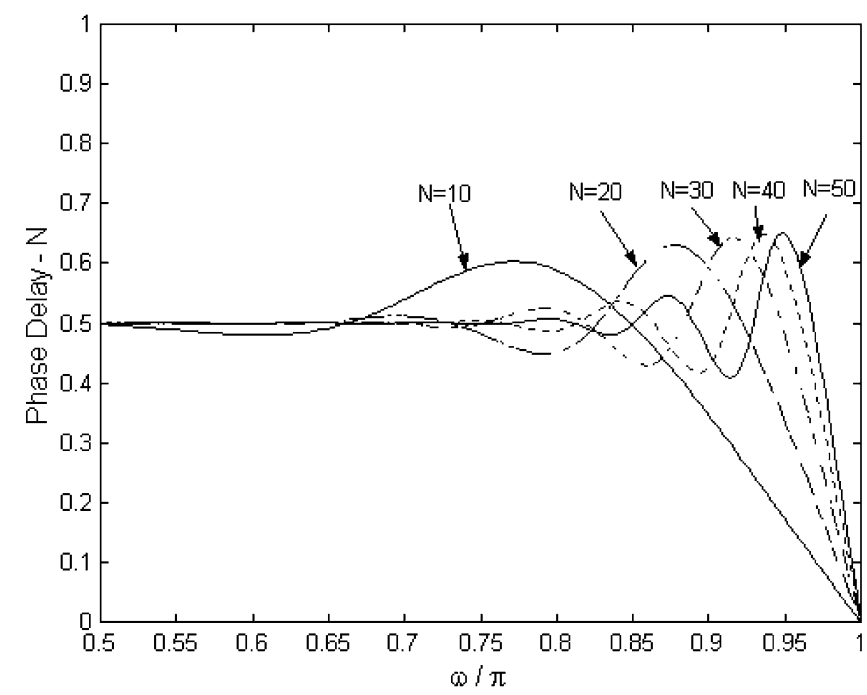

(b)

Fig. 3. Phase delay plots for $N=10,20,30,40,50$ over $0.5 \pi<\omega<\pi$. (a) $d=-0.5$. (b) $d=0.5$. The plot illustrates the error due to truncation of power series.

for $d<0$ are wider than the bandwidths for $d>0$. Fig. 3 illustrates the degradation of phase response due to truncation of power series. If the power series is truncated to $N$ terms, we obtain an $N$ th AP FD filters. The fraction part phase responses for $N=10,20,30,40,50$, and $d= \pm 0.5$ are shown in Fig. 3 . The width of the band of interest is increased if we use more terms for the approximation.

\section{CONCLUSIONS}

A new closed-form AP FD filter is proposed in this letter. The coefficients of the proposed filter are obtained by truncating the power series expansion of the function of $\left(1+z^{-1}\right)^{-d}$ expanded at $z^{-1}=1$. According our derivation in this letter, $\left(1+z^{-1}\right)^{-d}$ may be regarded as the ideal transfer function of denominator. Instead of the Schur-Cohn criterion or Jury-Marden criterion, we apply the Eneström-Kakeya theorem to prove the stability of the proposed AP FD filter with $0<d<1$. The closed-form expression of coefficients facilitates analysis of stability. On the other hand, the stability of filters with $-1<d<0$ is demonstrated by numerically computing the poles. We find that all the poles are inside unit circle if $-1<d<0$ for $N<100$. The proposed filters are degraded when the precision of the coefficient is low. According to our experiment, coefficients rounded to 8 bits or less will induce large ripples over $[0, \pi / 2]$. The ripples can be suppressed if 16 bits or more are used for expressing the coefficients.

\section{REFERENCES}

[1] T. I. Laakso, V. Välimäki, M. Karjalainen, and U. K. Laine, "Splitting the unit delay," IEEE Signal Processing Mag., vol. 13, pp. 30-60, Jan. 1996.

[2] A. V. Oppenheim, R. W. Schafer, and J. R. Buck, Discrete-Time Signal Processing, 2nd ed. Englewood Cliffs, NJ: Prentice-Hall, 1999.

[3] E. Hermanowicz, "Explicit formulas for weighting coefficients of maximally flat tunable FIR delayers," Electron. Lett., vol. 28, no. 20, pp. 1936-1937, Sept. 1992.

[4] S. C. Pei and P. H. Wang, "Closed-form design of maximally flat FIR Hilbert transformers, differentiators, and fractional delayers by power series expansion," IEEE Trans. Circuits Syst. I, vol. 48, pp. 389-398, Apr. 2001.

[5] Z. Jing, "A new method for digital all-pass filter design," IEEE Trans. Acoust., Speech, Signal Processing, vol. ASSP-35, pp. 1557-1564, Nov. 1987.

[6] J.-P. Thiran, "Recursive digital filters with maximally flat group delay," IEEE Trans. Circuit Theory, vol. CT-18, pp. 659-664, Nov. 1971.

[7] M. Makundi, T. I. Laakso, and V. Välimäki, "Efficient tunable IIR and allpass filter structure," Electron. Lett., vol. 37, no. 6, pp. 344-345, March 2001

[8] N. P. Murphy, A. Krukowski, and I. Kale, "Implementation of wideband integer and fractional delay element," Electron. Lett., vol. 30, no. 20, pp. 1658-1659, September 1994.

[9] Handbooks of Mathematical Functions, 9th ed., M. Abramowitz and I. A. Stegun, Eds., Dover, New York, 1972.

[10] A. Antoniou, Digital Filters: Analysis, Design, and Applications, 2nd ed. New York: McGraw-Hill, 1993.

[11] M. Marden, The Geometry of Zeros of a Polynomial in a Complex Variable. New York: Amer. Math. Soc., 1949.

[12] N. Anderson, E. B. Saff, and R. S. Varga, "On the Eneström-Kakeya theorem and its sharpness," Lin. Alg. Applicat., vol. 28, pp. 5-16, 1979. 